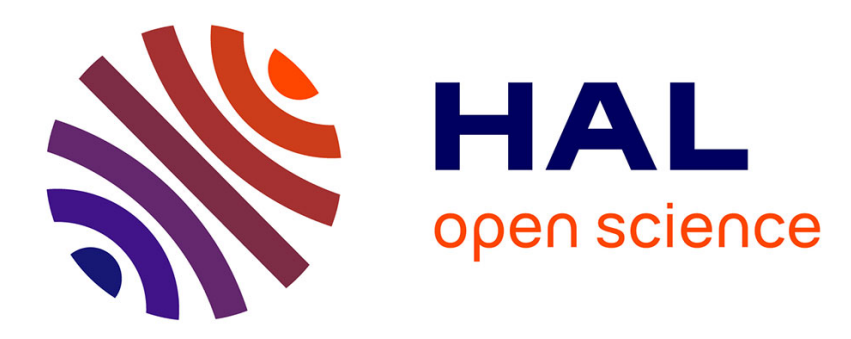

\title{
Des éphémères en devenir : les petites revues fin-de-siècle
}

Julien Schuh

\section{To cite this version:}

Julien Schuh. Des éphémères en devenir: les petites revues fin-de-siècle. Les éphémères: objets, corpus, culture (16e-21e siècles), Olivier Belin; Florence Ferran, Jan 2014, Paris, France. pp.35-44. hal-01140238

\section{HAL Id: hal-01140238 \\ https://hal.science/hal-01140238}

Submitted on 9 Apr 2015

HAL is a multi-disciplinary open access archive for the deposit and dissemination of scientific research documents, whether they are published or not. The documents may come from teaching and research institutions in France or abroad, or from public or private research centers.
L'archive ouverte pluridisciplinaire HAL, est destinée au dépôt et à la diffusion de documents scientifiques de niveau recherche, publiés ou non, émanant des établissements d'enseignement et de recherche français ou étrangers, des laboratoires publics ou privés. 


\section{DES ÉPHÉMÈRES EN DEVENIR : LES PETITES REVUES FIN-DE-SIÈCLE}

On ne rangerait pas a priori les revues littéraires dans la catégorie des éphémères : souvent publiées de manière soignée, sur beau papier, dans une mise en page qui appelle la reliure et la conservation, ces périodiques semblent relever davantage du monde du livre que de celui de la presse, dont le rythme de production et les méthodes de diffusion rendent la conservation plus aléatoire. En analysant ces objets, on constate cependant que toutes les parties d'une revue ne connaissent pas le même destin. Leur matérialité garde les traces de la multiplicité des enjeux esthétiques, symboliques, sociaux, commerciaux dont elles sont les vecteurs ; traces qui en font des objets polymorphes, hétéroclites, fragmentés et mobiles. Leurs couvertures, souvent oubliées lors de leur transformation en recueils, s'ouvrent à la réclame, aux correspondances, aux annonces ; des pages de publicité, de souscription, s'insèrent, sur des papiers et dans des formats différents du reste de la revue, entre des poèmes symbolistes et des estampes précieuses, elles-mêmes souvent conçues comme des parties détachables qui sont par conséquent souvent absentes des collections. La petite revue est un objet en tension, dont les divers éléments ne sont pas conservés de la même manière : tout ce qui relève de l'actualité, du médiatique, du commercial prend un caractère éphémère, qui s'oppose au devenir-livre du contenu artistique et littéraire. Elles remettent en cause aussi bien l'unité du livre que la pertinence des classifications traditionnelles.

Je prendrai l'exemple particulier des « petites revues » fin-de-siècle, pour reprendre l'expression de Remy de Gourmont ${ }^{1}$ : des périodiques publiés par de jeunes auteurs, de manière éphémère et en tirage limité, en marge des courants institués. Beaucoup de revues sont créées dans le dernier quart du $\mathrm{XIX}^{\mathrm{e}}$ siècle, profitant des conditions matérielles, politiques et économiques très favorables de la presse sous la Troisième République. Dans le contexte de la mise en réseau des pratiques littéraires à la fin du siècle, les écrivains d'avant-garde se distinguent de la littérature industrielle et de son système économique en instituant cercles, cénacles, en se constituant en groupes réunis autour de petites revues ${ }^{2}$.

On a pu contester à juste titre le caractère marginal de ces publications, dont certaines connaissent des tirages plus qu'honorables et survivent pendant plusieurs années, tendant de plus en plus à la pérennité du livre ${ }^{3}$. Mais la grande majorité de ces «petites revues » ou « revues de jeunes » ne connaît pas le succès de La Revue blanche ou du Mercure de France : publiées par des groupes de jeunes écrivains et artistes, parfois à peine sortis du lycée, le plus souvent autofinancées (chaque rédacteur paye une partie de la publication, qui s'arrête souvent, faute de lecteurs, lorsque les contributeurs se retrouvent sans le sou), ces revues n'ont souvent que quelques mois d'existence (comme Le Moderniste illustré d'Aurier en 1889). Leur périodicité aléatoire, leur manque de diffusion, leur tirage restreint en font des objets destinés à disparaître rapidement ; lorsque Remy de Gourmont entreprend en 1900 son catalogage des Petites revues, fondé sur sa propre collection, la plupart des périodiques qu'il décrit, et qui n'ont paru que quelques années auparavant, sont déjà quasiment introuvables. Le Mercure de France publie dès octobre 1891 une « Petite Tribune des Collectionneurs » en fin de numéro qui dresse la liste des ouvrages recherchés par des abonnés de la revue ; les Entretiens politiques et littéraires, La

\footnotetext{
${ }^{1}$ Remy de Gourmont, Les Petites Revues, essai de bibliographie, Paris, Librairie du Mercure de France, 1900. ${ }^{2}$ Voir Alain Vaillant, L'Histoire littéraire, Armand Colin, coll. Lettres U, 2011, p. 269 et 280 (le « système avantgardiste $\gg$ ).

3 Évanghélia Stead, « De la revue au livre : notes sur un paysage éditorial diversifié à la fin du XIXe siècle », Revue d'histoire littéraire de la France, vol. 107, 4/2007, p. 803-823.
} 
Vogue (en particulier les numéros consacrés à Rimbaud), La Revue indépendante, voire les premiers numéros du Mercure lui-même font partie des raretés convoitées par les amateurs ${ }^{4}$.

Si elles font aujourd'hui l'objet de recherches de plus en plus précises, pour elles-mêmes et non plus en tant que sources de prépublications ou d'anecdotes de l'histoire littéraire ${ }^{5}$, les petites revues restent encore d'un accès difficile : les numéros disponibles en bibliothèque sont extrêmement fragiles et font partie de fonds à accès restreint ; les collections sont incomplètes ou mal répertoriées. Les revues accessibles par le biais de numérisations sont le plus souvent les revues les plus connues, et même dans ce cas, leur présentation est souvent incomplète ${ }^{6}$; la numérisation de La Revue blanche dans Gallica est ainsi issue du reprint Slatkine, ce qui a entraîné la disparition de toutes les couvertures, où l'on trouve aussi bien des informations sur la vie de la revue que des annonces de publications, d'événements, des réclames, voire des comptes rendus ou des œuvres originales (fig. 1).

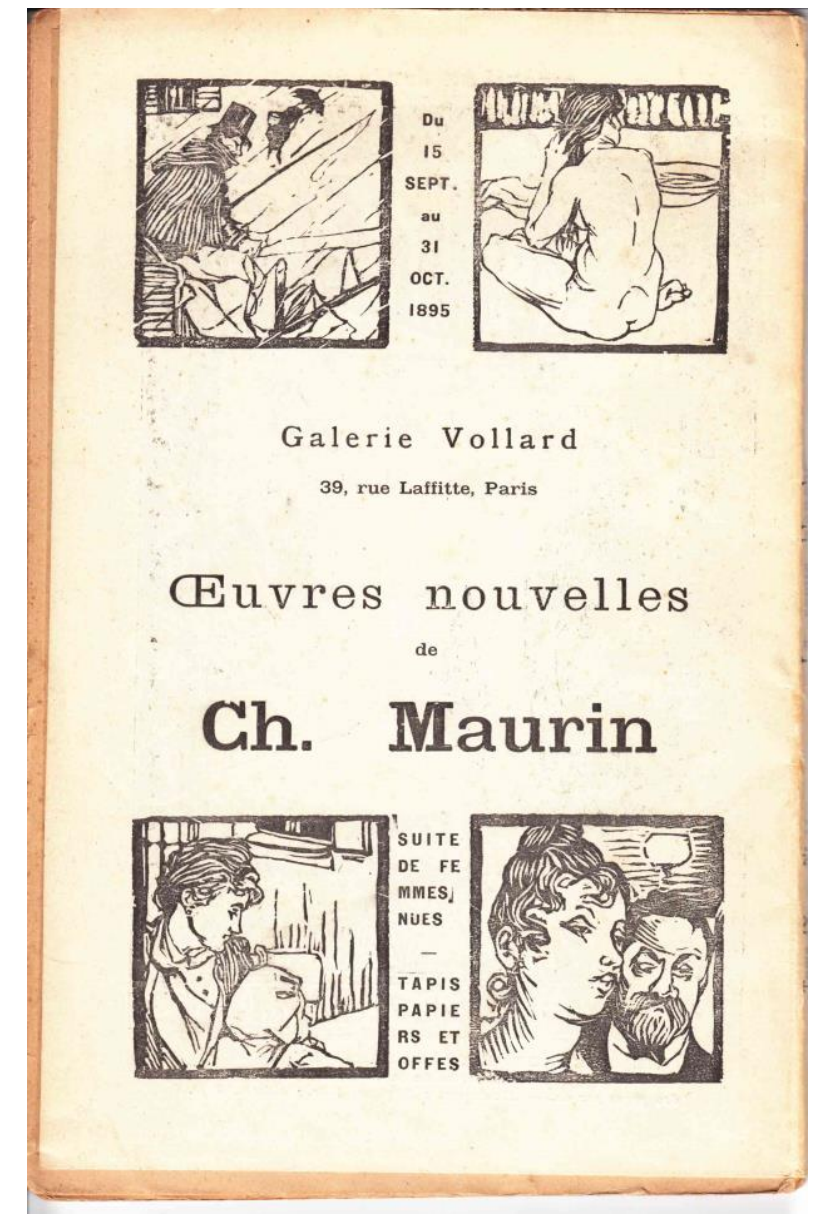

Fig. 1. Charles Maurin, gravure sur bois, quatrième de couverture de La Revue blanche, $\mathrm{n}^{\circ}$ 55, 15 septembre 1895. [coll. part.]

\footnotetext{
${ }^{4}$ Mercvre, «Petite Tribune des Collectionneurs », Mercure de France, t. III, n²2, octobre 1891, p. 255-256, URL : http://mercurewiki.fr

${ }^{5}$ Voir Évanghélia Stead \& Hélène Védrine (dir.), L’Europe des revues (1880-1920), estampes, photographies, illustrations, Paris, Presses de l'Université Paris-Sorbonne, 2008 ; Peter Brooker et Andrew Thacker (dir.), The Oxford Critical and Cultural History of Modernist Magazines, vol. I-III, Oxford, Oxford University Press, 2009-2013.

${ }^{6}$ Cette question a été abordée lors d'une demi-journée d'étude PATRIMA « Périodiques et Numérisation » organisée par Philip Leu à la BnF en mai 2012.
} 
Mais ce caractère éphémère des petites revues ne se limite pas aux difficultés de conservation ou à la rareté de certains exemplaires. Il est en réalité inscrit dans les modalités de consommation de ce type de publication périodique.

Il faut tout d'abord considérer les différences génériques entre les périodiques désignés par les termes de « petites revues », qui réunissent des objets aux conditions matérielles d'existence très diverses. Le Décadent ou Le Symboliste appartiennent davantage au genre des journaux, aussi bien par leur mise en page, leur périodicité que leur papier journal ${ }^{7}$. Une revue comme L'Art littéraire de Lormel publie en 1892 ses premiers numéros dans un format journalistique (deux colonnes, page de Une) avant de se transformer en revue sur le modèle du Mercure de France lorsqu'Alfred Jarry et Léon Fargue s'invitent dans le comité de rédaction en 1894 (avec couverture, pages de garde et sommaire fondés sur le modèle du livre ${ }^{8}$ ). À l'autre bout du spectre, Le Centaure avec Régnier, Jean de Tinan, Valéry, Gide, s'offre en 1896 sous la forme d'un livre relié de toile verte, comme le Yellow Book ${ }^{9}$. Une revue comme La Plume publie des réclames pour des dispositifs de reliure permettant aux amateurs de constituer leur propre recueil annuel, et propose également des reliures éditeurs annuelles, dont les sommaires complexes permettent aux lecteurs de se retrouver dans le riche contenu de la publication. On pourrait établir un classement de ces revues en les disposant dans un champ avec plusieurs pôles vers lesquels elles tendent plus ou moins (entre le livre et le journal d'une part, l'art et la réclame d'autre part). Le Centaure, avec ses gravures originales et sa reliure, tendrait vers la bibliophilie, tandis qu'une revue comme Le Canard sauvage, qui publie Jarry, Jules Renard, Vallotton, Franc-Nohain, tendrait plutôt vers le pôle de la revue satirique grand public, moins destinée à la conservation.

Mais les revues d'avant-garde de cette époque sont également des objets polymorphes, fragmentés dans leur conception et leur composition mêmes, et dont les différentes parties ne relèvent pas du même statut : couvertures, réclames, bulletins d'abonnements, hors-textes ne sont pas destinés à être utilisés ni conservés de la même manière. Cette hybridité constitutive des revues, dont l'unité apparente cache une fragmentation réelle, est d'autant plus difficile à concevoir qu'elle est évolutive : la forme de ces revues se modifie dans le temps de leur diffusion, de leur consommation et de leur conservation. Les pages centrales, allégées des couvertures, réclames et autres, sont réunies pour devenir des livres, au prix d'un effacement des marques précises de leur contexte de publication (quiconque a jamais tenté de feuilleter le recueil annuel d'une revue sans ses couvertures sait à quel point il peut être difficile de trouver la ligne de démarcation entre les différents numéros). Les réclames sont mises au rebut; les couvertures subissent souvent le même sort, à moins, dans le meilleur des cas, d'être recueillies en fin de volume ; les estampes finissent souvent accrochées sur un mur ou dans un portefeuille, ce qui fait enrager les collectionneurs (et les chercheurs) qui entassent des numéros privés de leurs gravures hors texte. Ce phénomène est progressif : chaque étape du destin d'une revue l'éloigne de sa forme primaire de diffusion (qui n'est que passagère, dans l'esprit même des

\footnotetext{
${ }^{7}$ Véronique Silva Pereira parle d'ailleurs à leur sujet de " petits journaux »; voir son article « "Les premières armes du symbolisme" : le rôle du "petit journal" dans la querelle symboliste de 1886 ", COnTEXTES, 11/2012, mis en ligne le 18 mai 2012. URL : http://contextes.revues.org/5318 ; DOI : $10.4000 /$ contextes.5318

${ }^{8}$ Patrick Fréchet, «L'Art littéraire : Fiche bibliographique », L'Étoile-Absinthe, nos 39-40, 1988, p. 29. URL : http://alfredjarry.fr/amisjarry/fichiers ea/etoile absinthe 039 40reduit.pdf

${ }^{9}$ Nathalie Froloff, «Le Centaure et ses modèles anglais : The Pageant et The Yellow Book », L'Europe des revues, op. cit., p. 295-311.
} 
rédacteurs de ces périodiques) pour contribuer à sa fragmentation. Les objets qui nous sont parvenus aujourd'hui sont souvent figés à des moments différents de ce processus : les numéros de revue isolés, tels qu'ils ont été diffusés, sont rares ; dans les bibliothèques, les reliures prédominent, obéissant (ou non) au découpage par volume prévu dans les tables des matières annuelles ou semestrielles distribuées dans les revues avec les couvertures ad hoc; les réclames et couvertures mensuelles sont souvent manquantes ; les estampes sont parfois conservées à part, dans des fonds iconographiques.
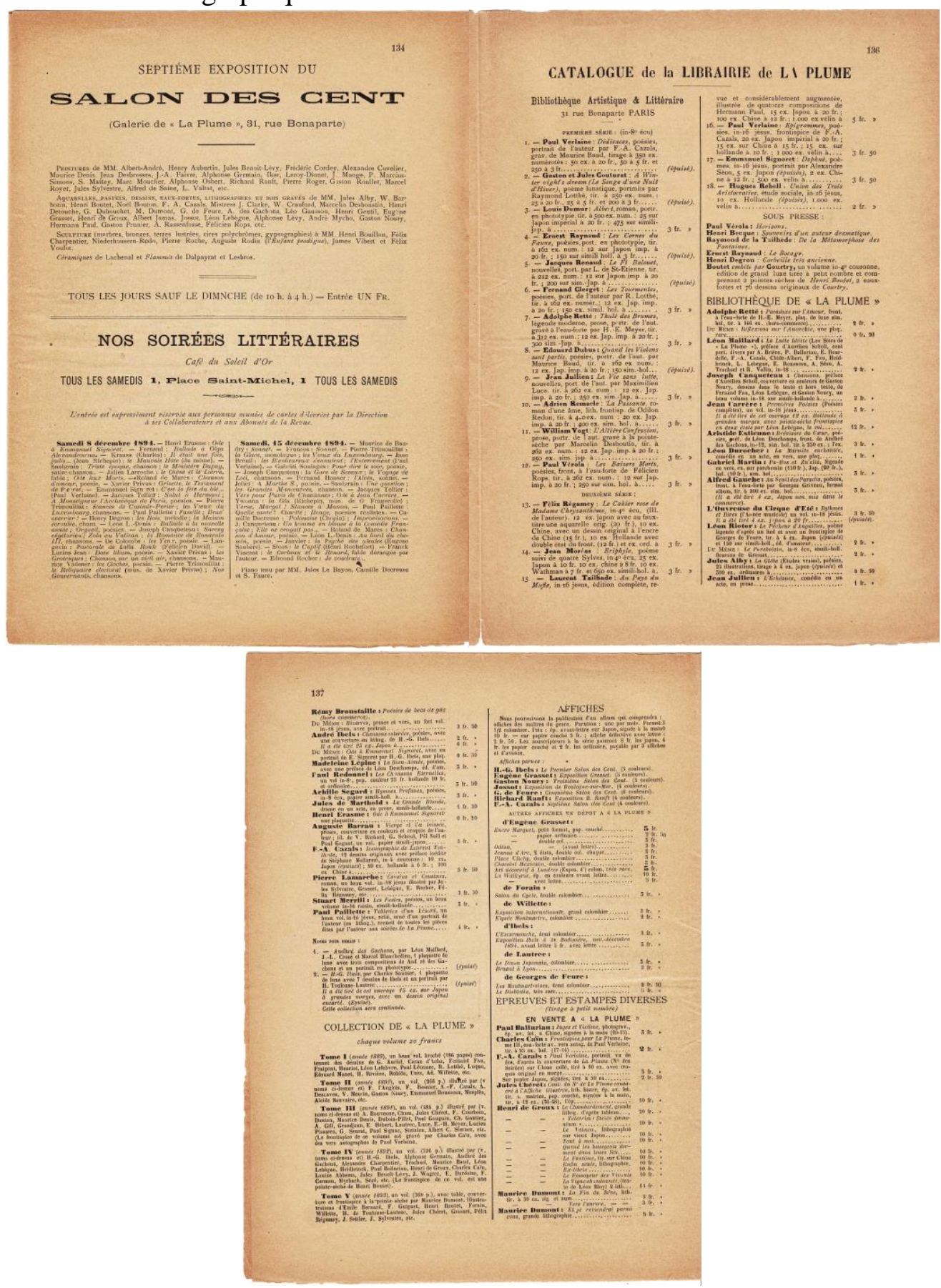

Fig. 2-4. Annonces d'exposition et de soirées, catalogues de librairie, d'estampes et d'affiches dans La Plume, ${ }^{\circ}$ 136, 15 décembre 1895, n.p. [coll. part.] 
Pour illustrer cette hétérogénéité générique et temporelle, je donnerai l'exemple d'un périodique particulièrement complexe, La Plume, revue éclectique, mêlant avant-garde et arts industriels (elle défend l'art de l'affiche ${ }^{10}$, organise le Salon des Cent présentant les artistes défendus par la revue), saluant aussi bien les artistes naturalistes que symbolistes. Cette revue est la vitrine d'un ensemble de pratiques (maison d'édition, galerie d'art, commerce de l'estampe et de l'affiche, organisation de soirées littéraires...) qui s'affichent dans les pages d'annonces de la revue, confirmant son rôle essentiel dans une nouvelle économie de l'avantgarde (fig. 2-4). Ces pratiques transparaissent également dans l'hétérogénéité de la maquette de la revue, qui fait coexister des éléments destinés à être conservés avec des pages relevant de l'éphémère.

Je ferai ressortir quelques observations de l'analyse du n 262 du 15 mars 1900 de ce périodique, consacré en partie à l'œuvre d'Eugène Grasset, un artiste particulièrement mis en avant dans la revue. L'une des particularités de La Plume est de publier de longs dossiers consacrés à des artistes en plusieurs fascicules ; ce numéro est le deuxième consacré à Grasset.

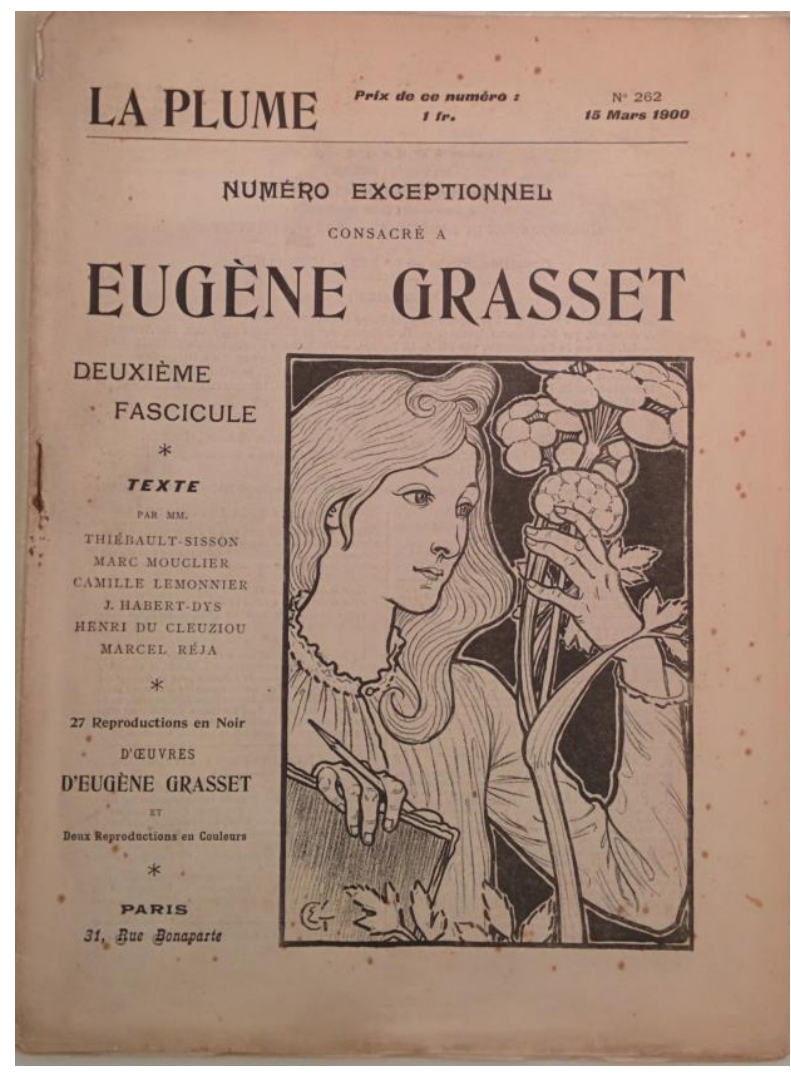

\section{Fig. 5. Première de couverture de $L a$ Plume,$n^{\circ}$ 262, 15 mars 1900. [coll. part.]}

La couverture elle-même (fig. 5) peut être considérée comme une forme d'éphémère : probablement imprimée dans un autre atelier que le corps de la revue, elle concentre les informations liées à l'actualité (banquets, publications, courrier des lecteurs, annonces diverses). Dans les premières années de La Plume, la couverture est imprimée sur des feuilles jaunes légères, d'un format légèrement plus réduit que les pages de la revue, ce qui lui donne

\footnotetext{
${ }^{10}$ Nicholas-Henri Zmelty, « Le discours critique de la revue La Plume sur l'affiche illustrée (1893-1899) », Image \& Narrative, $\mathrm{n}^{\circ}$ 20, " L'Affiche fin-de-siècle ", décembre 2007 [en ligne]. URL : http://www.imageandnarrative.be/inarchive/affiche findesiecle/zmelty.htm
} 
l'aspect d'une simple chemise destinée à protéger la revue le temps de sa diffusion, et dont on peut se débarrasser au moment de la relier dans un recueil annuel. Les rédacteurs prennent cependant rapidement conscience de l'importance des couvertures dans la vie de la revue, et $\mathrm{La}$ Plume présente la spécificité d'inclure les pages de couverture (avec leur propre système de numérotation) dans le sommaire annuel - ce qui rend d'autant plus regrettable leur absence dans la plupart des reliures en bibliothèque ou dans le reprint donné par Slatkine.

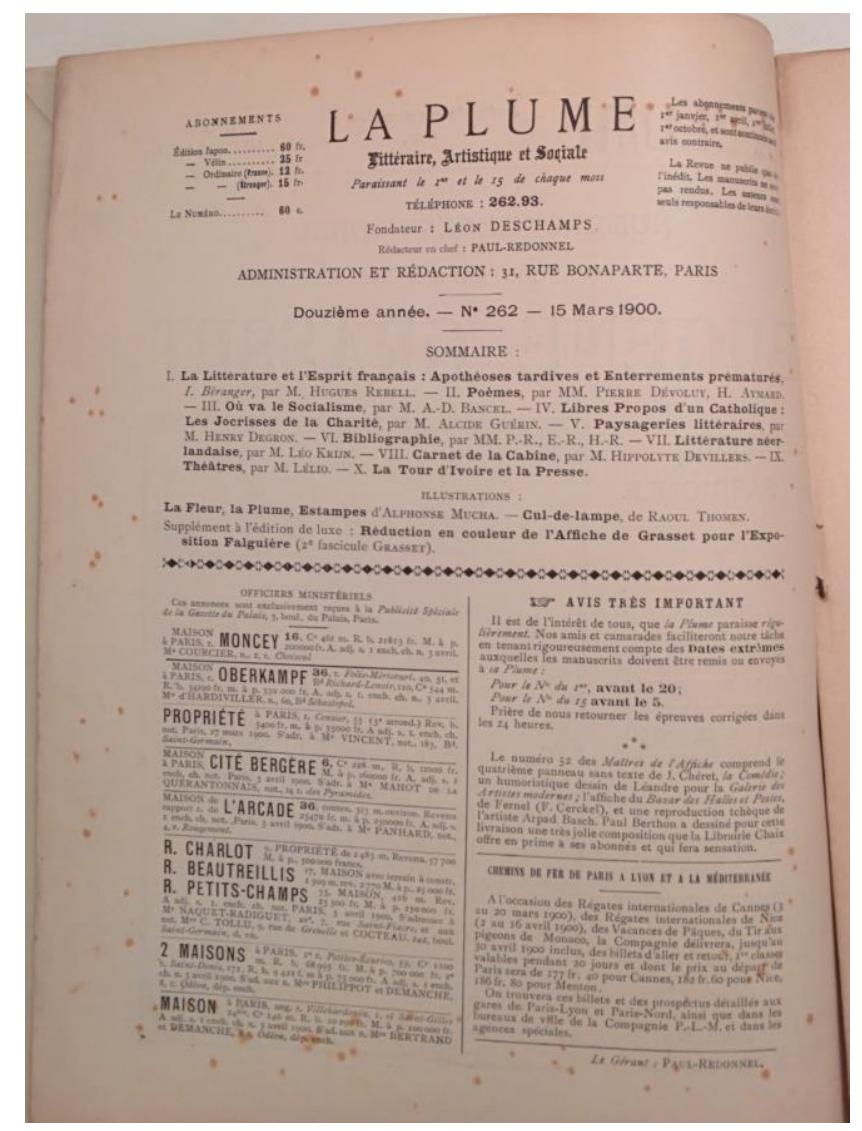

Fig. 6. Deuxième de couverture de $L a$ Plume, ${ }^{\circ}$ 262, 15 mars 1900. [coll. part.]

Un autre élément important risque de disparaître avec la couverture : le sommaire simplifié du numéro, en deuxième de couverture (fig. 6). Différent de celui qui est livré en fin d'année, il semble voué au rebut, entouré qu'il est de réclames, petites annonces, avis divers, comme le reste de la couverture, qui représente pourtant une sorte de nœud médiatique de la revue, fonctionnant comme une interface entre le public et la rédaction : son analyse permet de mesurer les transactions effectuées par la rédaction pour faire exister l'espace singulier de la revue au sein de l'espace social et du champ littéraire de son époque. 


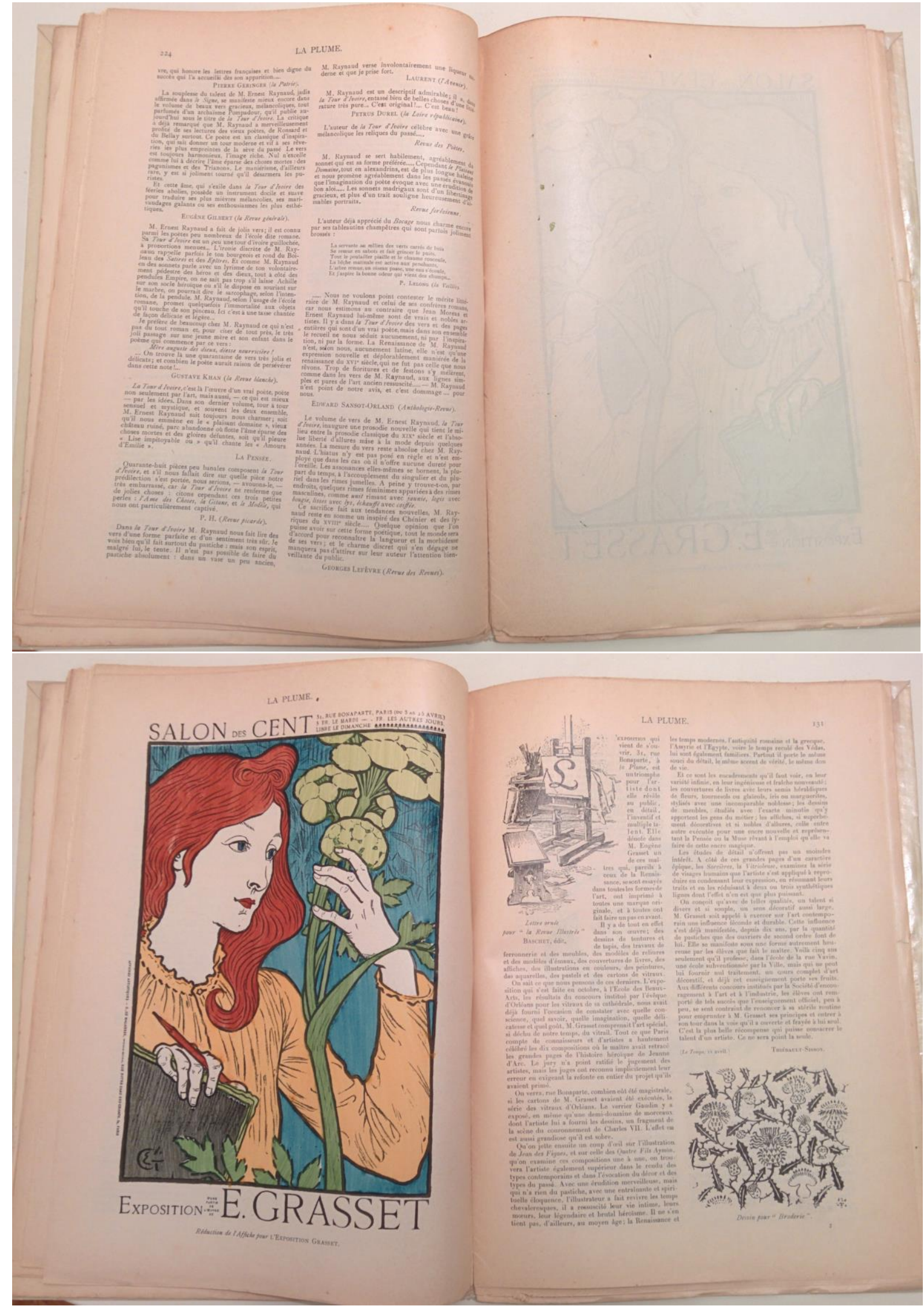

Fig. 7-8. Eugène Grasset, réduction de l'affiche du Salon des Cent, photogravure rehaussée au pochoir, La Plume, $\mathbf{n}^{\circ}$ 262, 15 mars 1900, n.p. [coll. part.] 
Les estampes hors-textes sont elles aussi appelées à un destin particulier. Ainsi de certaines illustrations de Grasset, coloriées au pochoir (fig. 7-8). On notera qu'elles sont imprimées à dessein au verso de pages vierges, ce qui permet de les détacher sans nuire à la cohérence du reste de la revue. La réduction d'une affiche du Salon des Cent consacré à Grasset publiée dans ce numéro, rehaussée au pochoir selon une méthode artisanale peu coûteuse (encore employée à cette époque par l'Imagerie d'Épinal), transforme cet objet reproduit par procédé photomécanique en estampe précieuse, avec ses imperfections de coloris qui la rendent unique et augmentent sa valeur pour les collectionneurs. Les premiers numéros de La Plume contenaient systématiquement le portrait d'un écrivain ou d'un artiste de la revue, imprimé sur une feuille glissée entre les pages, sur le modèle des portraits à collectionner dont l'époque était friande (avec la collection Félix Potin, les recueils de portraits de célébrités du vin Mariani, du chocolat Guérin-Boutron...).

La numérotation des pages qui entourent cette illustration est remarquable : on constate qu'on passe de la page 224 à la page... 131. En effet, le cahier qui précède l'illustration constitue le contenu habituel de la revue (poèmes, comptes rendus...), tandis que le cahier qui la suit ouvre le deuxième fascicule consacré à Grasset. Ce changement de pagination indique que les différents cahiers consacrés à Grasset doivent être reliés à la suite lors de la transformation des numéros de la revue en recueil annuel. Il y a donc une réorganisation programmée de la matière de la revue entre sa diffusion et sa conservation ; sans recours aux numéros isolés, il ne reste plus aucune trace de cette substitution dans la version " définitive » du volume conservé. C'est la composition même de la revue qui devient ici une forme d'éphémère : la matière du périodique a pu être conservée dans les recueils, mais son principe original d'organisation a disparu.

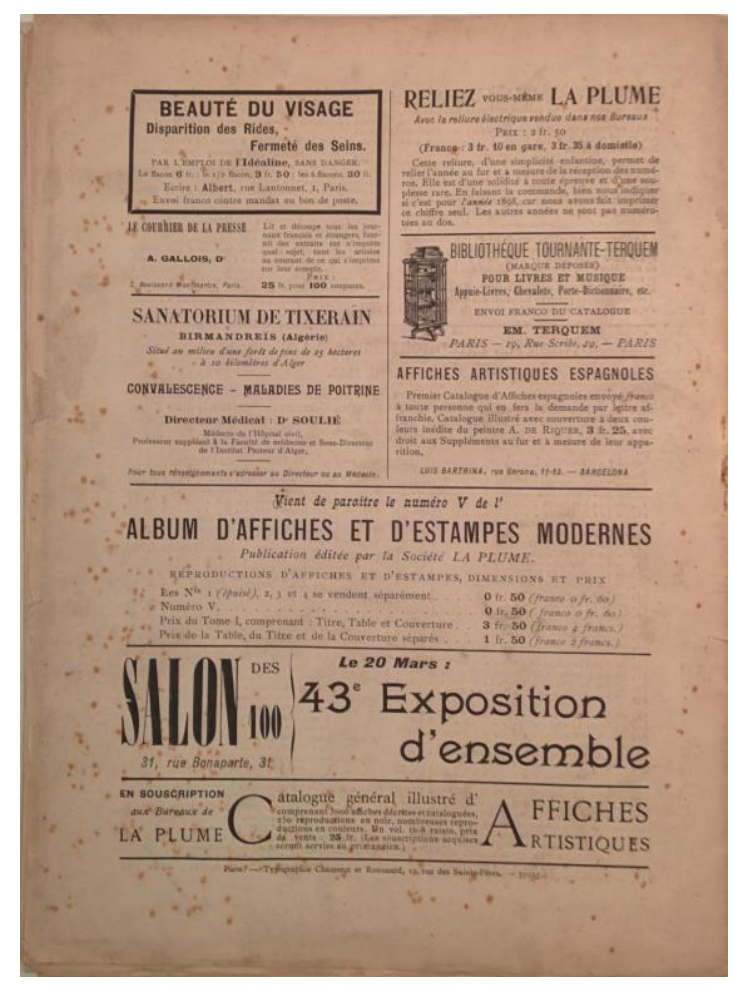

Fig. 9. Réclames, quatrième de couverture de La Plume, $\mathbf{n}^{\circ}$ 262, 15 mars 1900. [coll. part.] 
On notera enfin, en quatrième de couverture (fig. 9), diverses annonces (dont une réclame pour des reliures électriques : «Reliez vous-même La Plume »). Le traitement typographique, la mise en page, le choix du papier permettent de distinguer clairement tout ce qui relève de l'actualité, du commerce, mettant ces pages au même niveau que des prospectus de réclame.

Les petites revues proposent ainsi la réalisation de plusieurs destins possibles pour les imprimés, et ces modifications sont programmées dans les revues elles-mêmes. Elles relèvent à la fois des imprimés destinés à durer et des éphémères : on trouve en leur sein des catalogues (liste des publications de la maison d'édition, d'estampes à vendre), des petites annonces, des affiches (reproduites en réduction), des réclames (publiées le plus souvent sur des papiers plus légers), des bons de commande ou de réservation (par exemple pour Le Latin mystique de Gourmont dans le Mercure), des éléments relevant de l'actualité médiatique (comptes rendus théâtraux ou littéraires) qui se traduisent matériellement à travers des choix différents de papiers, de typographies, de mises en page. Ces objets sont destinés à se transformer dans le temps, à être diffusés sous plusieurs formes (numéros isolés, recueils), à être transformés pour être conservés - ce qui les rend extrêmement difficiles à classer selon les catégories traditionnelles de la bibliothéconomie.

Le caractère industriel de ces périodiques, publiés dans une ère de médias de masse, nous inciterait à considérer tous les exemplaires d'un même numéro de revue comme des objets identiques, dans une production parfaitement calibrée. Mais un certain nombre d'écarts remettent en cause cette manière de voir. L'analyse des couvertures des revues de cette époque permet de confirmer la non-uniformité des exemplaires conservés : Alexia Vidalenche, qui a étudié les couvertures de différents exemplaires d'un même numéro de La Revue blanche, a pu montrer que la typographie, les annonces et réclames des couvertures peuvent différer d'un exemplaire à l'autre du même numéro ${ }^{11}$. Si ces différences ne semblent pas calculées, on peut renvoyer à la revue Les Taches d'encre de Maurice Barrès (1884), dont chaque exemplaire est maculé de taches d'encre véritables qui le rend unique, ou au Petit Journal des Refusées, qui ne connut qu'un numéro à San Francisco en 1896 : son directeur, James Marrion, fit un usage délibéré de tampons de couleurs sur ses couvertures pour singulariser chaque exemplaire ${ }^{12}$. La disparition de ces couvertures de la plupart des exemplaires conservés et des numérisations disponibles efface des éléments essentiels de compréhension de ces périodiques.

Un exemplaire donné d'une revue de cette époque ne peut donc pas être considéré comme un représentant parfait de la totalité des exemplaires produits, un prototype dont la numérisation permettrait de conserver l'essentiel. Les petites revues doivent au contraire être analysées avec les mêmes outils que les livres anciens, avec une attention aux moindres détails de leur matérialité. Les multiples différences qui se glissent entre deux exemplaires d'un même numéro conservé (dans la typographie des couvertures, l'application des couleurs au pochoir, les erreurs d'impression, les papiers des différentes éditions disponibles, les choix de conservation...) doivent nous conduire à analyser chaque exemplaire comme une forme d'unica : un point dans une série avec des variations.

Julien Schuh

\footnotetext{
${ }^{11}$ Alexia Vidalenche, Couvertures et pages liminaires des revues fin-de-siècle : une avant-scène médiatique pour les groupes symbolistes (La Plume - La Revue blanche - L'Art littéraire - Le Livre d'Art), mémoire de Master II, Université de Reims Champagne Ardenne, 2014, p. 104-106.

12 Le Modernist Journals Project a numérisé trois exemplaires différents de cette revue. URL : http://www.modjourn.org/render.php?view=mjp object\&id=1183478160359375
} 